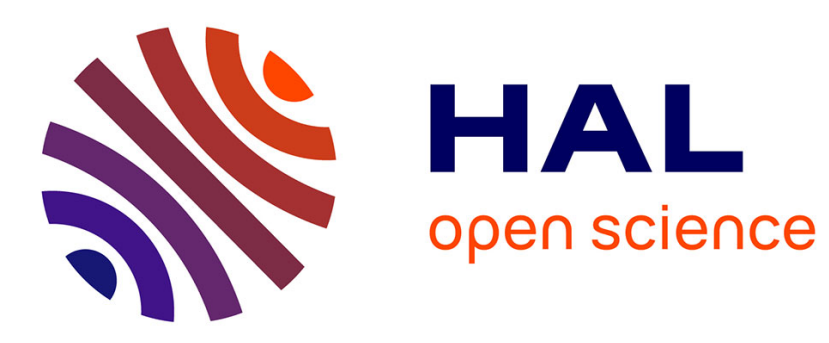

\title{
The spreading of macroscopic droplets
}

\author{
P. Levinson, A.M. Cazabat, M. A. Cohen Stuart, F. Heslot, S. Nicolet
}

\section{To cite this version:}

P. Levinson, A.M. Cazabat, M. A. Cohen Stuart, F. Heslot, S. Nicolet. The spreading of macroscopic droplets. Revue de Physique Appliquée, 1988, 23 (6), pp.1009-1016. 10.1051/rphysap:019880023060100900 . jpa-00245900

\section{HAL Id: jpa-00245900 https://hal.science/jpa-00245900}

Submitted on 1 Jan 1988

HAL is a multi-disciplinary open access archive for the deposit and dissemination of scientific research documents, whether they are published or not. The documents may come from teaching and research institutions in France or abroad, or from public or private research centers.
L'archive ouverte pluridisciplinaire HAL, est destinée au dépôt et à la diffusion de documents scientifiques de niveau recherche, publiés ou non, émanant des établissements d'enseignement et de recherche français ou étrangers, des laboratoires publics ou privés. 
Classification

Physics Abstracts

$68.10 \mathrm{G}-61.25 \mathrm{H}$

\title{
The spreading of macroscopic droplets
}

\author{
P. Levinson, A. M. Cazabat, M. A. Cohen Stuart (*), F. Heslot and S. Nicolet \\ Collège de France, Physique de la Matière Condensée, 11 place Marcelin-Berthelot, 75231 Paris Cedex 05, \\ France
}

(Reçu le 5 octobre 1987, révisé le 25 novembre 1987, accepté le 18 décembre 1987)

\begin{abstract}
Résumé. - Nous avons étudié à l'échelle macroscopique l'étalement sur des surfaces lisses de gouttes posées ou pendantes. Pour les gouttes posées, les résultats sont en bon accord avec les prédictions de la théorie de de Gennes et Joanny. En revanche, l'hypothèse de self-similarité utilisée dans cette théorie et approximativement valable pour les gouttes posées est grossièrement inexacte pour les gouttes pendantes pour lesquelles n'existe pas de traitement théorique. Nous proposons une résolution numérique des équations hydrodynamiques, qui n'utilise pas cette hypothèse et est donc valable aussi bien pour les gouttes posées que pour les gouttes pendantes. Nous supposons en revanche que les gouttes sont, à tout instant, en quasi équilibre et que par conséquent leur forme peut être calculée à l'aide de l'équation de Laplace. Nous avons d'autre part interprété des expériences d'étalement de gouttes posées sur des surfaces rugueuses aléatoires par un modèle simple, inspiré des théories de de Gennes et Joanny, qui explique bien les modifications induites par la rugosité dans la dynamique de l'étalement.
\end{abstract}

\begin{abstract}
Some experimental results on the macroscopic spreading of hanging and sessile drops on smooth surfaces are presented. The results for sessile drops nicely corroborate the main aspects of the spreading theory of de Gennes and Joanny. However, it is shown that one assumption of the theory, namely the retainment of a self-similar shape during spreading, which is approximately true for sessile drops, cannot be used for hanging drops, for which no theory is available. We propose a numerical resolution of the hydrodynamic equations which relaxes the necessity of self-similarity. The calculation involves the assumption that the shape of a (sessile or hanging) drop at any given time is in quasi-equilibrium with itself and can therefore be calculated through the Laplace equation. The calculation is indeed capable of describing the spreading of both sessile and hanging drops in detail. Spreading of sessile drops on rough surfaces may also be interpreted in the spirit of the theory of de Gennes and Joanny. Evidence is presented that the kinetics of the macroscopic foot which develops at the edge of a drop spreading on a rough surface is related to the heterogeneous distribution of the macroscopic contact angle and obeys simple equations.
\end{abstract}

\section{Introduction.}

Spreading of oil drops on surfaces is by no means a simple phenomenon, obeying simple equations $[1$, 2]. It is easy to understand that drops of apolar liquids will spread on most surfaces as the spreading parameter $S\left(\equiv \gamma_{\mathrm{SV}}-\gamma_{\mathrm{SL}}-\gamma\right)$ will often be positive. However, due to the change in shape of a drop during spreading, the appropriate hydrodynamic equations are very involved.

A more simple problem is that of a liquid edge

(*) Permanent address : Agricultural University, Laboratory for Physical and Colloid Chemistry, De Dreijen 6, 6703 BC Wageningen, The Netherlands. advancing with constant velocity $U$ over a solid surface. Some years ago, Hoffman [3] found that in that case and for $S>0$ the macroscopic contact angle is a function of the capillary number $\mathrm{Ca}(\equiv \eta U / \gamma)$ only, and not, as one might expect, of the spreading parameter $S$. For low capillary numbers the experimental relation between $\theta$ and $\mathrm{Ca}$ was found to be [4]

$$
\theta^{3} \simeq 3 C a
$$

In this article, we will summarize our recently obtained experimental and theoretical results concerning the spreading of silicone oil droplets on glass surfaces. We will focus our attention to the underly- 
ing physical principles more than to the details of the experiments. Our final aim will be to find the limits of validity of the theory of de Gennes and Joanny, and study how it can be used to deal with still more complicated situations.

Theory.

1. STATIONARY ADVANCING LIQUID EDGE. - Some years ago, Tanner [4] derived equation (1) theoretically for the particular case $S=0$ (Eq. (1) is also known as Tanner's law). Next, de Gennes and Joanny $[5,6]$ showed that Tanner's law is also valid for $S>0$. A positive $S$ does not interfere with the spreading dynamics of the macroscopic drop because a very thin precursor film develops in front of the macroscopic edge. In the film, $S$ is entirely burnt up by viscous dissipations $[5,6]$.

The width of the crossover region $\left(x_{1}\right)$ between the macroscopic edge and the film is of the order of $\sim a / \theta^{2}$ (where $a \sim \AA$ ) and thus extremely small (see Fig. 1).

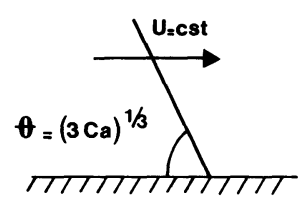

Macroscopic

(a)

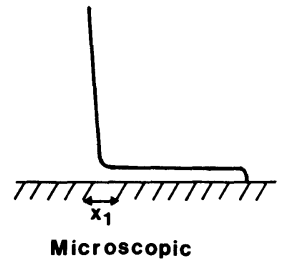

(b)
Fig. 1. - Liquid edge at macroscopic (a) and microscopic (b) scales.

2. NON-STATIONARY SPREADING OF NON-VOLATILE LIQUIDS. - As stated in the introduction, the hydrodynamic equations describing the change of shape of a spreading droplet are involved [5, 6]. However, they can be solved approximately by introducing the so-called self-similar assumption [5]. In this context, self-similarity means that the height $\xi(r, t)$ of the drop at position $r$ and time $t$ is given by $\xi=f(t) \cdot z(r / R)$, where $f$ is a function of time only and $R$ the radius of the wetted spot. It is easy to see that self-similarity automatically implies that the product $h(t) R(t)^{2}$ (where $h(t) \equiv \xi(0, t)$ ) is constant during spreading for non-volatile liquids.

An important parameter is the ratio of the radius $R$ to the capillary length $\kappa^{-1}\left(\equiv(\gamma / \rho g)^{1 / 2}\right)$. For $\kappa R \ll 1$, the gradient of the Laplace pressure near the edge is the sole driving force. The shape of the drop is then a spherical cap, slightly deformed in the crossover region $x_{1}$ (Fig. 2).

For $\kappa R \gg 1$, gravitation drives the spreading. The drop is then not spherical but more flattened near the centre. Nevertheless, as in the previous case, at

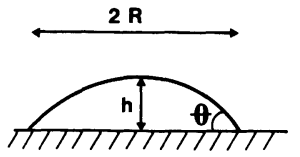

(a)

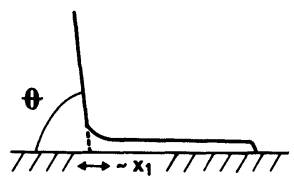

(b)
Fig. 2. - Drop's edge at macroscopic (a) and microscopic (b) scales.

every moment the shape of the drop is in quasiequilibrium, except very close to the edge.

The theory [5,6] predicts that, if $R(t) \gg x_{1}$,

$$
\begin{array}{ll}
R(t) \sim \Omega^{3 / 10}(\gamma t / \eta)^{1 / 10} & \kappa R \ll 1 \\
R(t) \sim \Omega^{3 / 8}(\rho g t / \eta)^{1 / 8} & \kappa R \gg 1
\end{array}
$$

with a scaling crossover for $\kappa R \sim 1$ ( $\Omega$ is the volume of the drop).

Equation (2a) can also be derived more directly from Tanner's law. For $\kappa R \ll 1$, the law may be interpreted as the balance between the capillary force $F_{\mathrm{c}}$ at the drop edge and the friction force $F_{\text {v }}$

$$
\mathrm{F}_{\mathrm{c}}=\frac{1}{2} \gamma \theta^{2} \quad(\theta \ll 1) .
$$

Within logarithmic factors, $F_{\mathrm{v}}$ can be written as :

$$
F_{\mathrm{v}} \sim \frac{\eta}{\theta} \frac{\mathrm{d} R}{\mathrm{~d} t}
$$

Combination of equations (3a) and (3b) results in

$$
\theta^{3}(t) \sim \frac{\eta}{\gamma} \frac{\mathrm{d} R}{\mathrm{~d} t}
$$

which is just Tanner's law, with $\mathrm{d} R / \mathrm{d} t$ replacing $U$. Furthermore, for $\kappa R \ll 1$ and $\theta \ll 1$, the shape of the drop is approximately a spherical cap, so that we have the additional relations

$$
\begin{aligned}
& \theta(t) \simeq \frac{2 h(t)}{R(t)} \\
& \Omega(t) \simeq \frac{\pi}{2} R(t)^{2} h(t)=\mathrm{Cst} .
\end{aligned}
$$

which are the low $\theta$ limits of the usual formulae

and

$$
\begin{aligned}
\operatorname{tg} \theta & =\frac{2 h}{R-\frac{h^{2}}{R}} \\
\Omega & =\frac{\pi}{2} R^{2} h\left[1+\frac{3 h^{2}}{2 R^{2}}\right] .
\end{aligned}
$$

Inserting these relations in equation (4) yields equation $(2 \mathrm{a})$.

For large drops things are slightly more complicated. Clearly, in this case the gravitational force $F_{\mathrm{g}}$ is much larger than $F_{\mathrm{c}}$. In addition, the viscous 
force $F_{v}$ will not depend on the contact angle, but on the ratio $R / h$ as the drop is considerably flattened,

$$
\begin{aligned}
& F_{\mathrm{g}} \sim \rho g h^{2} \\
& F_{v} \sim \eta \frac{R}{h} \frac{\mathrm{d} R}{\mathrm{~d} t} .
\end{aligned}
$$

Combination of equations (6a ) and (6b) gives

$$
\frac{\rho g h^{3}}{\eta} \sim R \frac{\mathrm{d} R}{\mathrm{~d} t}
$$

Next, we must supplement equation (7) by an additional relation between $h$ and $R$. Inserting the self-similar approximation $h(t) R^{2}(t)=$ Cst yields equation (2b).

\section{Results.}

SPREADING OF OIL DROPLETS. - Many experimental studies on the spreading of silicone oil drops (usually polydimethylsiloxane) have already been published [4, 7]. Silicone oil is convenient because it has a low surface tension $\left(\gamma=20.6 \mathrm{mN} \cdot \mathrm{m}^{-1}\right)$, and therefore wets most surfaces and is non-volatile. In addition extreme clean surfaces are not required to obtain reproducible results at the macroscopic scale. Moreover, a wide range of viscosities is available.

Most of these studies dealt with small drops $(\kappa R \ll 1)$ and the results were in good agreement with the predicted power laws $\Omega^{3 / 10} t^{1 / 10}$. We decided to study larger drops around the crossover range $R \sim \kappa^{-1}$. For silicone oil $\kappa^{-1}=1.48 \mathrm{~mm}$. The radius $R$ of the wetted spot was recorded versus time for drop volumes between 0.4 and $40 \mu \mathrm{l}$. Low viscosity oils $(0.02 ; 0.1 ; 0.5 ; 1 \mathrm{~Pa} . \mathrm{s})$ were used as models for simple, non-volatile liquids. Complementary measurements with more viscous oils $(10 ; 20$; $100 \mathrm{~Pa} . \mathrm{s})$, for which polymeric effects could arise, were also performed.

1. Low viscosity oils. - As our studies of the spreading characteristics of low viscosity PDMS sessile drops have already been published [8], we shall only present a brief summary of the main results : usually, the data were collected for spreading time in the range of $30 \mathrm{~s}$ to $30 \mathrm{mn}$. At times shorter than $30 \mathrm{~s}$, transient phenomena associated with fairly large contact angles, were observed. For times larger than $30 \mathrm{~s}$, all the transients have vanished, and $\theta$ is small enough for the Tanner's law to hold.

In a $\log R$ versus $\log t$ plot, a good fit of the experimental points could be obtained by two successive straight lines, the first one with slope $1 / 10$, the second with slope $1 / 8$. The fit is significantly poorer with only one straight line with intermediate slope, which suggests that the transition between the capillary $\left(R \sim t^{1 / 10}\right)$ and gravity $\left(R \sim t^{1 / 8}\right)$ domains is rather sharp, at least for the variation [8] of $R$ versus $t$. Far from the transition, equations (2) were nicely corroborated.

The location of the transition between capillary and gravity domains for various drops volume in the $\log R$ versus $\log t$ diagram is the same for all the oils. However, it is not a line of constant $\log R$, which would have been expected from the theoretical analysis [8]: as a matter of fact, the predicted crossover corresponds to $R \sim \kappa^{-1}$. Various combinations of the radius $R$, the height $h$ (or the curvature radius at the apex $\mathcal{R}$ ) and the viscosity $\eta$ are able to describe the observed transition line. They are empiric combination which do not deserve discussion. We concluded only that in the crossover more than one characteristic length exists, i.e. that self-similarity does not hold, which is not surprising. But the presence of the viscosity was unexpected. It suggests that in the crossover some characteristic time for the change in the drop shape might play a role in the spreading process [9].

2. High viscosity oils. - Complementary measurement on sessile drops of higher viscosities $(10 ; 20$; $100 \mathrm{~Pa} . \mathrm{s})$ were also performed in the volume range of $0.4-40 \mu \mathrm{l}$. It is known that for small drops $(\kappa R \ll 1)$ the $t^{1 / 10}$ law is well obeyed [4] [10], i.e., no specific polymeric slipping effect is observed.

For larger drops, an unexpected behaviour was found. For $\eta=10 \mathrm{~Pa} . \mathrm{s}$, the experimental curves $\log R$ versus $\log t$ are very similar to those of the low viscosity oils with the usual transition line at the usual place. For $\eta>20 \mathrm{~Pa} . \mathrm{s}$, the transition is no longer visible, i.e., a good fit of the experimental points is obtained by a single straight line whose slope increases regularly from 0.10 for $\Omega \leqslant 1 \mu$ to 0.125 for $\Omega \geqslant 40 \mu$ l. This is illustrated in figure 3 , where the experimental points for the silicone with viscosity $\eta=100 \mathrm{~Pa} . \mathrm{s}$ are reported. The silicone polydispersity cannot be invoked to explain this smoothing of the curves because the transition was found not to depend on the oil in the $\log R$ versus Log $t$ frame.

Again, some characteristic time for the changes in drop shape might cause this effect.

For the largest $(\Omega>30 \mu l)$ and smallest $(\Omega<2 \mu l)$ drops, equations (2) are again obeyed :

The experimental accuracy is very good for the time variation (see Fig. 3). The agreement is poorer for the exponents in the $\Omega$ and $\eta$ dependency (10\% for the first one, $15 \%$ for the second one) but they are clearly different for $\kappa R \gg 1$ or $\kappa R \ll 1$. Note that longer times are needed for the transients to vanish ( $5 \mathrm{~min}$ for $\eta=100$ Pa.s).

3. Hanging drops. - For small, non-extended drops ( $\kappa R \ll 1$ ), the same behaviour is expected both for sessile and hanging drops because it is the range where gravity is negligible. 


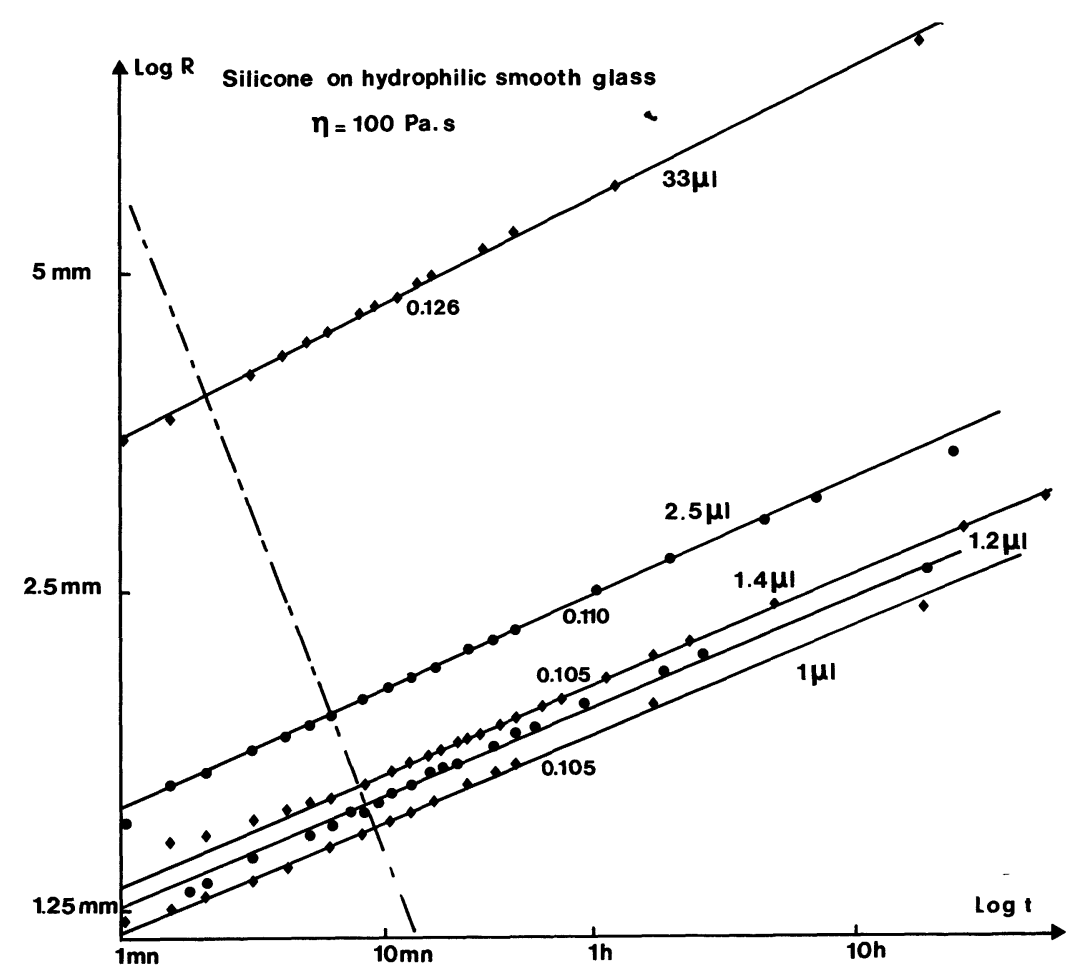

Fig. 3. - Log/Log plot of drop radius versus time for silicone oil of viscosity $\eta=100$ Pa.s (sessile drops). The slope of the straight line is indicated for each drop volume. The dashed line is the location of the transition between the capillary regime (slope 1/8) and the gravity regime (slope 1/10) for the oils of viscosity $<20 \mathrm{~Pa}$.s.

In the case of larger hanging drops $(\kappa R \geq 1)$, different phenomena were observed. Heavy drops $(\Omega \geq 59 \mu$ ) fall from the surface. Lighter ones first spread, then stop at a radius $R_{0}$ which was found to be practically independent of the drop weight, at least for $\Omega \geq 1 \mu$ l. We studied a series of drops of viscosity $0.5 \mathrm{~Pa}$.s with volumes in the range $1-20 \mu \mathrm{l}$.
The corresponding $\log R$ versus $\log t$ plots are given on figure 4 .

A good fit is obtained by single straight lines with slopes decreasing smoothly from 0.1 for small drops $(\Omega<4 \mu l, t<3 h)$ to 0.06 for $\Omega=21 \mu l$. At much longer times ( $t \geq$ day), all drops finally stop at $R_{0} \sim 6 \mathrm{~mm}$ and $\theta \simeq 0$. The value of $R_{0}$ is not precise

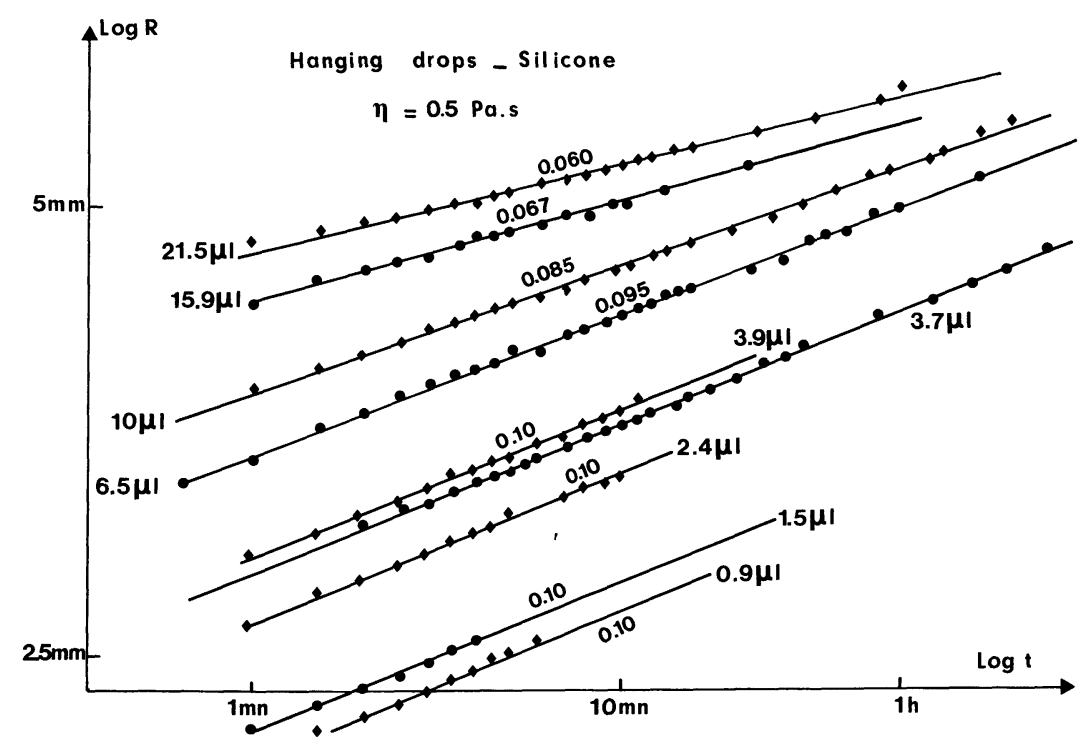

Fig. 4. - Log/Log plot of radius of hanging silicone drop versus time. Oil viscosity $\eta=0.5$ Pa.s. The slope of the straight line is indicated for each drop volume. 
because even the slightest (unintentional) tilt angle of the surface with the horizontal plane causes a significant slipping of the drop after several days.

Obviously, a finite value of the final radius does not correspond to true thermodynamic equilibrium because the spreading parameter is positive. At the edge of the drop a precursor film continues to grow, as for example in the case of a liquid meniscus in contact with a vertical wall $[5,11]$. However, the full development of the film would take many years.

At the time scale of our experiments, a quasi macroscopic equilibrium is reached with $\theta \simeq 0$ at the edge.

As soon as gravity plays a role, an approximate self-similar treatment is no longer adequate because the shape of the drop changes significantly during spreading. As yet, no prediction exists for this case.

We decided to investigate numerically the shape of the drops in order to obtain a description which would not involve any self-similarity assumption.

A NUMERICAL STUDY. - Previous studies [4, 5] have shown that a spreading drop has its static form (with a contact angle equal to the instantaneous dynamic contact angle $\theta(t)$ ) almost everywhere, except very close to the edge, and also that the dynamics of spreading is controlled by the macroscopic instantaneous contact angle $\theta$ according to the Tanner's law :

$$
\theta(t)^{3} \approx 3 \frac{\eta}{\gamma} \frac{\mathrm{d} R}{\mathrm{~d} t}
$$

The principle of the theoretical treatment is to calculate, for a series of given drop's volumes, the radius $R$ of a static drop as a function of its contact angle $\theta$. Standard techniques have been developed for this purpose [12]. The change of radius with time is calculated afterwards by using Tanner's law. It is assumed that in all cases $x_{1} \ll \kappa^{-1}, R$.

1. Basic formula. - The hydrostatic equilibrium condition for the balance of the vertical forces on a drop of volume $\Omega$ can be written [12] by using Laplace's law :

$$
\Omega=\pi R^{2}\left[h-\frac{2 \sin \theta}{R} \varepsilon \kappa^{-2}+\frac{2}{\mathfrak{R}} \varepsilon \kappa^{-2}\right]
$$

$\theta, h$ and $R$ have their usual meaning. $\mathfrak{R}$ is the absolute value of the curvature radius at the apex. $\varepsilon=+1$ for sessile drops and -1 for hanging drops.

- For $\kappa R \ll 1$, both sessile and hanging drops are spherical caps. For small $\theta, \frac{h}{R \theta} \rightarrow \frac{1}{2}$ and $\frac{R^{2} h}{\Omega} \rightarrow \frac{2}{\pi}$, i.e. self-similarity is achieved.

- For $\kappa R \gg 1$, sessile drops become cylinders of radius $R$. For small $\theta, \frac{R^{2} h}{\Omega} \rightarrow \pi$. Self-similarity is achieved again but with a different value for $\frac{R^{2} h}{\Omega}$

- For $\kappa R \gg 1$, no simple relation can be written for hanging drops.

2. Numerical results for small $\theta$. - The calculated plots $\log R$ versus $\log \theta$ are presented in figure 5 for both sessile and hanging drops. The rectangle represents the range of the experimental investigations.

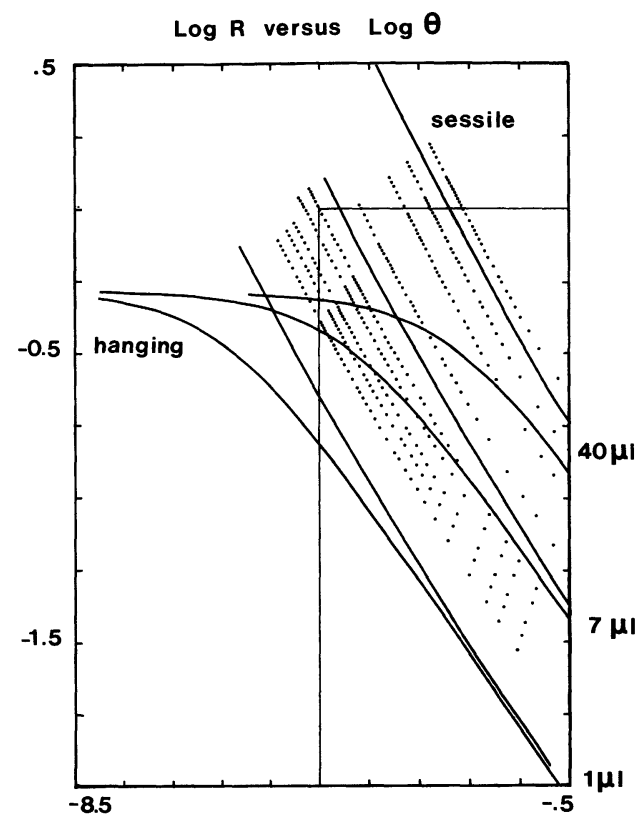

Fig. 5. - Calculated Log/Log plot of radius of sessile or hanging drops versus contact angle $\theta$, for various drop volumes. The range of experimental studies is located inside the (lower right) rectangle and corresponds to $1 \mu l \leq \Omega \leq 40 \mu l$. The points are the calculated values. For clarity, only those of the sessile drops have been plotted. The whole continuous curves for $\Omega=1 \mu l$, $7 \mu l, 40 \mu l$ have also been drawn.

The curves associated with sessile drops become slightly steeper at low angles (upper left). In contrast, those corresponding to hanging drops flatten and tend towards a constant value for very low contact angles. In the lower right part of the diagram, i.e. for $\kappa R \ll 1$, the curves associated with sessile and hanging drops of given volume become identical. The common limiting slope of the curves in this range is $\alpha=-\frac{1}{3}$.

If the corresponding relation $R \sim \theta^{-1 / 3}$ is combined with the Tanner's law $\theta^{3} \sim \frac{\mathrm{d} R}{\mathrm{~d} t}$, the expected variation $R \sim t^{1 / 10}$ is found.

For sessile drops and $\kappa R \gg 1$ (upper left), the slopes of the curves tend toward another limit, $\alpha=-\frac{3}{7}$, slightly different from the preceding one. 
Inserted in Tanner's law, this leads to $R \sim t^{1 / 8}$, in good agreement with the predictions of the selfsimilar approximation. (It is not obvious from figure 5 that $\alpha=-\frac{3}{7}$ is the limiting slope at vanishing $\theta$. However, additional calculations for $\Omega=40 \mu \mathrm{l}$ and smaller angles did not indicate any further change in the slope). However the validity of the numerical calculation, assuming $x_{1}$ to be negligible, is restricted to $\theta \geq 10^{-3}$.

Finding a good agreement with the predictions of the self-similar approximation for $\kappa R \ll 1$ and $\kappa R \gg 1$ is not surprising because self-similarity holds in these limiting cases (Fig. 6). But both figures 5

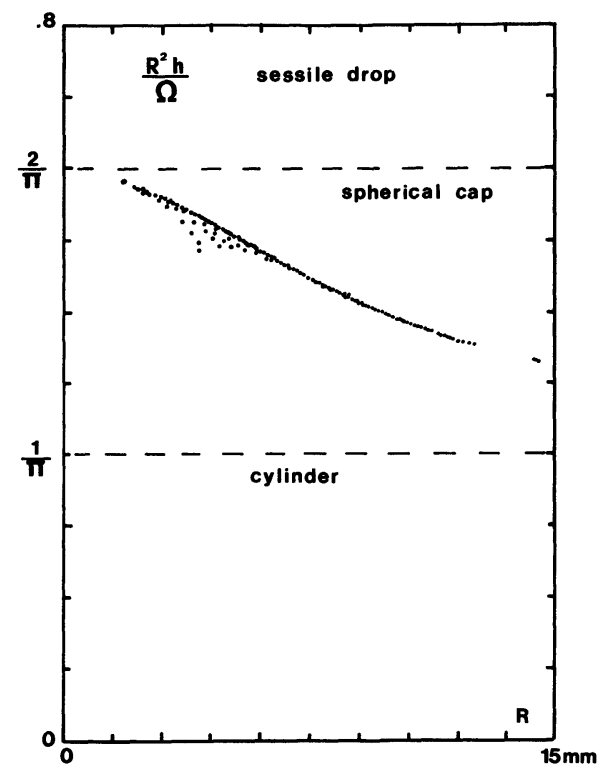

Fig. 6. - Calculated values of $h R^{2} / \Omega$ versus $R$ for sessile drops. The upper envelope of the points corresponds to the low $\theta$ limit. (Points below this envelope have large $\theta$ values, for which the approximation $\operatorname{tg} \theta \approx \sin \theta \approx \theta$ does not hold.)

and 6 show that the crossover is rather wide. The spreading of high viscosity oils (smooth experimental curves, Fig. 3) is better described than the spreading of low viscosity ones (experimental sharp transition). The shape of the drops for the latter case might be different from the static one in the crossover.

From the numerical results, we can predict the apparent (mean) slope of the various curves of the figure 3. The agreement is quite good, indicating that the numerical results satisfactorily describe the drop behaviour.

For hanging drops, the slope of the $\log R$ versus $\log \theta$ plot goes to zero at vanishing $\theta$. A constant value $R_{0} \approx 7.5 \mathrm{~mm}$, practically independent of the drop volume in the investigated range, is obtained for $\theta \rightarrow 0$.

In this limit, the calculated value of $\frac{R^{2} h}{\Omega}$ is

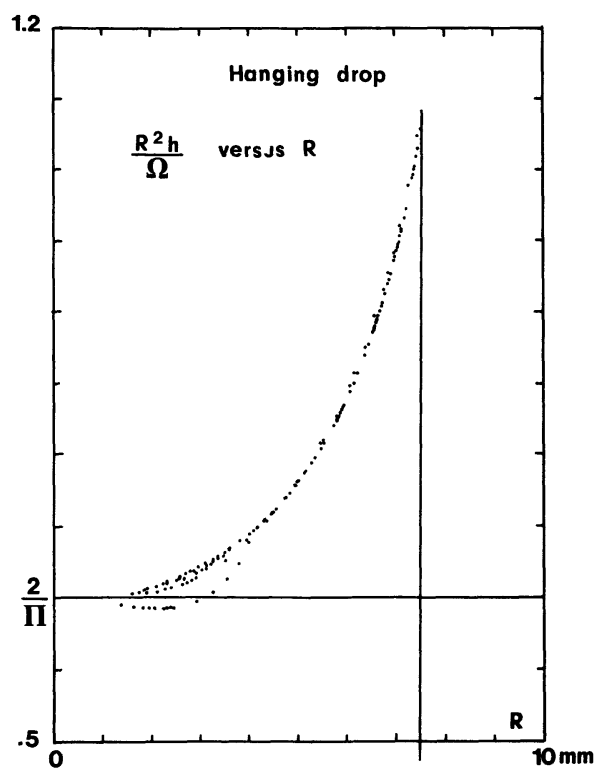

Fig. 7. - Calculated values of $h R^{2} / \Omega$ versus $R$ for hanging drops. The upper envelope of the points is again the low $\theta$ limit.

$\approx 1.1$ (see Fig. 7). For the volume $\Omega$ of the drop, we have (insert $\theta=0$ in Eq. (8))

$$
\Omega(\theta=0)=\pi R^{2} h\left[1-\frac{2 \kappa^{-2}}{h \mathfrak{R}}\right] .
$$

As $\frac{R^{2} h}{\Omega}$ is 1.1 for $\theta=0$, the product $\Re h$ is a constant, independent of the drop volume. In the same limit, $R$ is also a constant $R=R_{0}$. If we compare drops for different volumes at $\theta=0$, they can be deduced by a similarity in the vertical direction (Fig. 8).

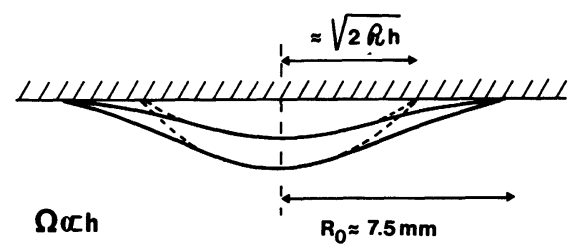

Fig. 8. - Schematic behaviour of hanging drops with various volumes, stopped at $R=R_{0}(\theta=0)$.

Then the behaviour of hanging drops in the $\theta \rightarrow 0$ limit is explained. Again in this case, there is a good agreement between the expected slopes and the experimental ones for the curves in figure 4.

Moreover, a semi-empirical argument may describe the temporal relaxation of $R$ towards the asymptotic value $R_{0}$. For $\theta \rightarrow 0$ the calculated relation between $R$ and $\theta$ is approximately linear, with slope $s(\Omega)$,

$$
R_{0}-R(\theta) \approx s(\Omega) \theta \quad \text { for } \quad \theta \rightarrow 0 .
$$


Combining this approximate theoretical relation with Tanner's law, one obtains :

$$
R_{0}-R(t) \approx\left[\frac{3 s^{3} \eta}{2 \gamma t}\right]^{1 / 2} \text { for } \quad t \rightarrow \infty
$$

a result which is similar to that obtained for the relaxation of a macroscopic meniscus towards its equilibrium height [5].

An empirical expression for the slope $s$ as a function of $\Omega$ is found to be

$$
s(\Omega) \sim R_{0} \frac{\Omega_{0}^{2 / 3}-\Omega^{2 / 3}}{\Omega^{2 / 3}} \quad \Omega_{0} \approx 40 \mu \mathrm{l}
$$

so that the drop volume is expected to be an important parameter for the dynamics. Further experiments with improved horizontality control are needed to confirm these semi-empirical predictions.

As a conclusion, the numerical study is quite fruitful : it is able to treat satisfactorily the asymptotic situations where approximate self-similar solutions are available. Moreover, it is able to follow the drop's behaviour in the crossover ranges where these solutions do not hold. As these crossovers appear to be rather wide (they represent in fact practically the whole domain which can be experimentally investigated), it is important to have a description of the system in this case, at least in order to estimate the accuracy of the approximate self-similar treatment.

Up to now, we have only considered the spreading of drops on smooth, flat surfaces. Real surfaces always possess some degree of roughness, whose effect on the spreading process must be investigated.

SPREADING ON ROUGH SURFACES. - The present discussion is not a restatement of the general question of spreading on surfaces with various roughnesses, but it concerns a particular case where the physics is especially simple. It will also be short, because a more detailed report is available in the literature [13].

1. The rough surfaces. - The surfaces we used were depolished glass surfaces with various roughness amplitudes. The roughness profile is random, but with rather well defined slope, independent of the roughness amplitude, i.e., there is geometrical similarity between different samples.

The roughness amplitude $e$ was between 2 and $50 \mu \mathrm{m}$, the absolute slopes approximately $30^{\circ}$ with respect to the horizontal. So the drops were much larger than the characteristic scales of the roughness and only an averaged effect was expected on the spreading dynamics.

We tried to model this effect by a constant (per unit length) driving force $F_{\mathrm{d}}=\gamma \delta^{2}$ where $\gamma$ is the liquid vapour interfacial tension, as before. $\delta^{2}$ is an empirical factor accounting for the roughness, i.e. representing some mean squared, value of the slope and thus independent of roughness amplitude $e$, as long as the drop was thicker than $e$.

2. The physical process. - The spreading on rough surfaces presents analogies with the spreading on smooth surfaces when the precursor film is considered, although the length scales are different :

* at short times, the instantaneous contact angle $\theta$ is fairly large. The precursor film, whose length growths as $\theta^{-3}[5]$ is still very short.

For surfaces which are not too rough, $\theta^{2}$ will be much larger than $\delta^{2}$ and the roughness will have no effect on spreading;

* at longer times, a significant precursor film, driven by the constant force $S$ per unit length, has developed on smooth surfaces.

At a different scale, a foot with thickness $\approx e$ appears at the edge of the drop on the rough surface. The foot is connected to a reservoir (the macroscopic drop) and driven by the constant force per unit length $F_{\mathrm{d}}$.

In the limit where $\frac{\mathrm{d} L}{\mathrm{~d} t} \gg \frac{\mathrm{d} R}{\mathrm{~d} t}$ we have

$$
\gamma \delta^{2} \sim \eta \frac{L}{e} \frac{\mathrm{d} L}{\mathrm{~d} t}
$$

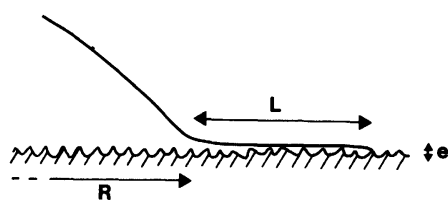

Fig. 9. - Drop spreading on a rough surface with well separated cap and foot.

The result is

$$
L(t) \sim\left(\frac{F_{\mathrm{d}} e}{\eta} t\right)^{1 / 2}
$$

which has the form of a diffusion equation.

In the limit of very extended precursor films, their thickness is also practically a constant $e_{\mathrm{c}}$ and the velocity at the film edge is much larger than the velocity at the drop edge. The precursor is driven by the constant force $S$ per unit length and one has [11]

$$
L_{\text {precursor }}(t) \sim\left(\frac{S e_{\mathrm{c}}}{\eta} t\right)^{1 / 2}
$$

i.e. also a diffusion-like equation;

* at very long times, the central drop disappears and our analogy fails. The behaviour of a flat drop on a smooth surface has been calculated by Joanny [5]. Here, we are still in the macroscopic domain and we therefore must incorporate the volume 
conservation condition. The variable is the drop radius

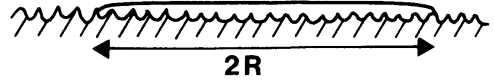

Fig. 10. - Flat drop spreading on a rough surface : The separation between cap and foot is no longer visible.

and one gets

$$
\begin{aligned}
\gamma \delta^{2} & \sim \frac{R}{h} \frac{\mathrm{d} R}{\mathrm{~d} t} \\
\text { or } \quad R(t) & \sim\left(\frac{F_{\mathrm{d}} \Omega}{\eta} t\right)^{1 / 4} .
\end{aligned}
$$

3. Comparison with experiment [13]. - This very crude model accounts fairly well for the observed dynamical laws :

- at short times, the laws for smooth surfaces $\left(t^{1 / 10}\right.$, then $\left.t^{1 / 8}\right)$ were found for silicone oils of low viscosity ;

- at intermediate times, the length of the foot is expected to obey :

$$
L(t) \sim e^{1 / 2} \eta^{-1 / 2} t^{1 / 2} .
$$

The agreement is very good for the $\eta$ and $t$ variations [13]. It is also good for $e$ in the range $e \geqslant 5 \mu \mathrm{m}$. But for lower roughnesses $L$ goes very rapidly to zero and becomes unobservable for $e<\mu \mathrm{m}$. We have no clear explanation for this behaviour;
- at longer times (but for drop thickness larger than $e$ ) the $t^{1 / 4}$ power law has been satisfactorily observed.

This brief discussion on rough surfaces was presented as an example of the use of simple models for problems which are unaccessible to mathematical exact models.

A practical interest of such experiments is also to recognize the range of roughnesses which will influence the spreading dynamics at the macroscopic scale $(e \geq \mu \mathrm{m})$.

\section{Conclusion.}

In this paper we have presented simple, macroscopic experiments on spreading dynamics. On smooth surfaces, the self-similar treatment by de Gennes and Joanny accounts satisfactorily for the asymptotic regimes of spreading and gives an approximate description of the crossover in the case of sessile drops. A comparison has been made with numerical calculations.

On rough random surfaces, the dynamics of spreading may be very different. New regimes of spreading may occur, which can be empirically accounted for by simple models.

These macroscopic studies are a perequisite to the experiments performed at the microscopic scale.

\section{Acknowledgments.}

Fruitful discussions with P. G. de Gennes, J. F. Joanny, H. M. Princen and H. J. M. Fraaye are gratefully acknowledged.

\section{References}

[1] Derjaguin, B. V., Zorin, Z. M., Churaev, N. V., ShISHIN, V. A., Wetting spreading and adhesion, Ed. Padday (Ac. Press) 1978.

[2] Dussan, E. B., Ann. Rev. Fluid Mech. 11 (1979) 371.

[3] Hoffman, R., J. Colloid Interface Sci. 50 (1975) 228.

[4] Tanner, L., J. Phys. D 12 (1979) 1473.

[5] Joanny, J. F., Thèse Le Mouillage, Paris (1985).

[6] De Gennes, P. G., Rev. Mod. Phys. 57 (1985) 827.

[7] Marmur, A., Adv. Colloid Interface Sci. 19 (1983) 75.

[8] Cazabat, A. M., Cohen Stuart, M. A., J. Phys. Chem. 90 (1986) 5845.
[9] Rosenblat, S., Davis, S. H., Frontiers in fluid mechanics, Ed. Springer (1985) pp. 171.

[10] Ausserré, D., Picard, A. M., Léger, L., Phys. Rev. Lett. 57 (1986) 2671.

[11] Joanny, J. F., DE Gennes, P. G., J. Phys. France 47 (1986) 121

[12] Princen, H. M., Surface and colloid science, Ed. E. Matijević (Wiley) 2 (1969) 1.

[13] Cazabat, A. M., Cohen Stuart, M. A., Colloid Polym. Sci. (1987) in press. 